\title{
Effects of a 'Baby-Friendly Hospital Initiative' on exclusive breastfeeding rates at a private hospital in Lebanon: an interrupted time series analysis
}

\section{Adrienne Clermont}

Cornell University Joan and Sanford I Weill Medical College

Josianne El Gemayel

Clemenceau Medical Center

Rola Hammoud

Clemenceau Medical Center

Jiangxia Wang

Johns Hopkins University Bloomberg School of Public Health

Hortenzia Beciu

Johns Hopkins Medicine

Mona Sinno

Clemenceau Medical Center

Wilma Berends

Johns Hopkins Medicine

Nadine Rosenblum

Johns Hopkins University School of Medicine

Jessica Bienstock

Johns Hopkins University School of Medicine

Kristen Byrnes

Johns Hopkins University School of Medicine

Roger Samuels ( $\nabla$ rwsamuels@jhmi.edu )

Johns Hopkins University School of Medicine https://orcid.org/0000-0002-4987-2585

Research article

Keywords: Breastfeeding, Lebanon, Maternity Care, Neonatal Care, Quality Improvement

Posted Date: September 1st, 2020

DOI: https://doi.org/10.21203/rs.3.rs-64827/v1 
License: (c) (i) This work is licensed under a Creative Commons Attribution 4.0 International License. Read Full License

Version of Record: A version of this preprint was published at BMC Pregnancy and Childbirth on May 19th, 2021. See the published version at https://doi.org/10.1186/s12884-021-03816-3. 


\section{Abstract}

Background: Exclusive breastfeeding (EBF) through six months of age has been scientifically validated as having a wide range of benefits, but remains infrequent in many countries. The WHO/UNICEF BabyFriendly Hospital Initiative (BFHI) is one approach to improve EBF rates.

Methods: This study documents the implementation of BFHI at Clemenceau Medical Center (CMC), a private hospital in Lebanon, and analyzes data on EBF practices among CMC's patients before, during, and after the implementation period. The process of launching the BFHI at CMC is discussed from the perspective of key stakeholders using the SQUIRE guidelines for reporting on quality improvement initiatives. As an objective measure of the program's impact, 2,002 live births from July 2015 to February 2018 were included in an interrupted time series analysis measuring the rates of EBF at discharge prior to, during, and following the bundle of BFHI interventions.

Results: The steps necessary to bring $\mathrm{CMC}$ in line with the BFHI standards were implemented during the period between November 2015 and February 2016. These steps can be grouped into three phases: updates to hospital policies and infrastructure (Phase 1); changes to healthcare staff practices (Phase 2); and improvements in patient education (Phase 3). The baseline percentage of EBF was $2.4 \%$ of all live births. Following the BFHI intervention, the observed monthly change in EBF in the "Follow-Up" period (i.e., the 24 months following Phases 1-3) was significantly increased relative to the baseline period (+2.0 percentage points per month, $p=0.006$ ). Overall, the observed rate of EBF at hospital discharge increased from $2.4 \%$ to $49.0 \%$ of all live births from the first to the final month of recorded data.

Conclusions: Meeting the BFHI standards is a complex process for a health facility, requiring changes to policies, practices, and infrastructure. Despite many challenges, the results of the interrupted time series analysis indicate that the BFHI reforms were successful in increasing the EBF rate among CMC's patients and sustaining that rate over time. These results further support the importance of the hospital environment and health provider practices in breastfeeding promotion, ultimately improving the health, growth, and development of newborns.

TRIAL REGISTRATION: Data collection procedures and protocols were reviewed by the Johns Hopkins Medicine Office of Human Subjects Research Institutional Review Board (application \#IRB00155989), and determined to not constitute human subjects research under DHHS or FDA regulations.

\section{Background}

CONTRIBUTIONS TO THE LITERATURE: 
- The Baby-Friendly Hospital Initiative (BFHI) is a 10-step, evidence-based program developed by WHO and UNICEF to improve practices at hospitals providing maternity care.

- Although evidence is mixed as to which components of the BFHI package are most important, systematic reviews indicate that healthcare-based interventions are effective in improving exclusive breastfeeding (EBF) rates, an important indicator for infant health outcomes.

- This study describes the implementation of a BFHI package at Clemenceau Medical Center in Beirut, Lebanon, and shows a significant and sustained improvement in EBF rates (from $2.4 \%$ to $49 \%$ ), demonstrating the effectiveness of this intervention in a Middle Eastern context.

\section{Problem Description and Available Knowledge}

The practice of exclusive breastfeeding (EBF) of newborn infants through six months of age has been validated as having a wide range of health benefits for both baby and mother $(1,2)$, as well as broader economic and environmental benefits for society (3). It is recommended as the standard for infant feeding by the World Health Organization (WHO) (4), the American Academy of Pediatrics (1), the American College of Obstetricians and Gynecologists (5), and the Lebanese Ministry of Health (6). However, despite knowledge of these benefits within the scientific and clinical community and nearly three decades of initiatives to promote breastfeeding, EBF rates remain low in many areas of the world (3). A variety of factors - including sociocultural attitudes, workplace and government policies, marketing of breastmilk substitutes, and entrenched clinical practices - contribute to lowered levels of EBF, particularly in higher-income populations and countries (3). Accordingly, the translation of EBF principles into general healthcare practice represents an important and evolving target for structured efforts at behavioral change within healthcare organizations.

Lebanon is an upper-middle-income country that is known to have low levels of sustained EBF. Although more recent nationally representative survey data are not available, a 2006 national survey found that while almost all mothers (95.4\%) initiated breastfeeding, by one month, only $52.4 \%$ of babies were exclusively breastfed (7). This declined further to $23.4 \%$ at four months and only $10.1 \%$ at six months. EBF was found to be inversely associated with urban residence and educational level of the mother. $A$ number of studies have examined the causes of low EBF rates in Lebanon, and have identified hospitalrelated factors (such as lack of rooming-in) (7); maternal employment and related barriers (8-11); negative perceptions of breastfeeding (fear of weight gain and breast sagging) and experiences of breastfeeding (insufficient milk, pain, sleep deprivation) (9); cultural beliefs around "bad milk" and potential harm to the infant (12); and lack of government policy engagement to promote breastfeeding ( 9 , 13).

Hospitals and healthcare providers are an important avenue for the promotion of EBF to new mothers. The Baby-Friendly Hospital Initiative (BFHI) was developed by the WHO and UNICEF in 1991 (updated in 2009 and 2018) as a "global effort to implement practices that protect, promote, and support breastfeeding" (14-16). It is comprised of a 10-step, evidence-based program to improve policies, training, and practices at hospitals and clinics providing maternity care. Since its inception, the BFHI 
program has been implemented in over 20,000 health facilities in 152 countries worldwide $(14,17)$. One recent systematic review found a positive relationship between implementation of the BFHI program and improved breastfeeding outcomes, with a dose-response between the number of BFHI steps to which women were exposed and the likelihood of EBF (18), while another systematic review found the heterogeneity in study designs and settings too high to draw an overall conclusion on BFHI's effectiveness (19). Recent critical re-evaluations of BFHI have suggested that focusing on specific evidence-based interventions for breastfeeding may be more important than adherence to the overall package or the BFHI certification itself (20). In the Middle East region in particular, studies have documented the effectiveness of BFHI implementation in hospitals in Saudi Arabia (21) and the United Arab Emirates $(22,23)$.

\section{Rationale and Specific Aims}

This study describes the experience of Clemenceau Medical Center (CMC), located in Beirut, Lebanon, with the implementation of the BFHI program in 2015. It analyzes data on EBF practices from 2,002 live births from July 2015 to February 2018 at CMC. This paper has two objectives: first, to describe the development and execution of an EBF program at CMC in the context of the BFHI model, using the SQUIRE guidelines for reporting quality improvement initiatives (24); and second, to conduct a quantitative statistical evaluation of the impact of BFHI practices on EBF rates at discharge using interrupted time series analysis.

\section{Methods}

\section{Setting and Context}

$\mathrm{CMC}$ is a 158-bed Joint Commission International ( $\mathrm{JCl}$ )-accredited private hospital in Beirut, Lebanon, with approximately 750 deliveries annually. $\mathrm{CMC}$ has an active affiliation with Johns Hopkins Medicine International $(\mathrm{JHI})$, through which a number of collaborations have been undertaken to promote quality and patient safety improvement, education, and strategic planning initiatives across several of CMC's hospital divisions. One such collaborative initiative, launched at CMC in 2015, was an effort to establish the standards outlined in the BFHI in order to respond to the challenges of low regional EBF rates.

\section{Intervention}

Beginning in October 2015, medical and administrative staff at CMC, with support from partners at JHI, implemented a series of actions to more systematically align CMC's policies and practices with those proposed by the BFHI. The process was spearheaded by the office of the Chief Quality Officer at CMC and included a core team of midwives, nurses, and physicians, as well as technical support from Johns Hopkins subject matter experts in obstetrics, lactation nursing, and project management. The implemented reforms can be grouped into three phases: updates to hospital policies and infrastructure (Phase 1); changes to healthcare staff practices (Phase 2); and improvements in patient education (Phase 3). The key interventions are summarized in Table 1, and a more detailed list of interventions with a timeline, grouped according to the "10 Steps" of the BFHI process, is available in Additional File 1. 
Table 1

Summary of key interventions in BFHI process at CMC

\section{Category}

Phase 1:

Hospital policies and

infrastructure

Phase 2:

Healthcare staff practices

Phase 3:

Patient education

\section{Key interventions}

- Completion of WHO self-assessment tool

- Revision of existing newborn policy

- Drafting of new policies on EBF, skin-to-skin contact, and rooming-in

- Revision of existing "no formula on the wards" policy

- Construction of a private breastfeeding room for consultations and patient use

- Formation of an EBF team to lead BFHI implementation process

- Incorporation of EBF topics into institutional training for new hires and current staff

- Implementation of rooming-in and skin-to-skin practices with patients

- Allocation of expert lactation consultant to supervise midwife/nurse team

- Addition of newborn flow sheet to electronic medical record to document feedings

- Requirement of medical order by pediatrician for an infant to receive formula

- Addition of EBF session into prenatal education classes for expecting mothers

- Provision of informational materials on breastfeeding and infant care at discharge

- Availability of lactation consultant for post-discharge consultations five days a week

- Creation of breastfeeding support phone line for questions 24 hours a day

The first phase of interventions pertained to aligning CMC hospital policies with the model outlined by the BFHI standards proposed by the WHO and UNICEF. After completing the "WHO Self-Assessment Tool," which is intended to assess a health facility's status prior to instituting BFHI, the team at CMC, in collaboration with the $\mathrm{JHI}$ team, revised existing policies and drafted several new ones to reflect a commitment to the adoption of evidence-based BFHI practices across the institution. This ensured the buy-in of the hospital leadership and provided a policy framework for the subsequent adjustment of practices. In parallel, infrastructure improvements intended to facilitate breastfeeding were implemented: 
the "nursery" area was closed for renovations and subsequently reopened with its name changed to "breastfeeding room" in order to encourage rooming-in (see below).

The second phase encompassed the implementation of programs designed to improve healthcare staff practices. Led by a newly created "EBF Team" (composed of midwives, nurse educators, and physicians) that championed the process, a variety of changes were instituted that made breastfeeding an integral part of the prenatal and postnatal care teams' work. Per WHO guidelines, EBF-related topics were incorporated into training for all new and existing CMC staff (clinical and non-clinical), and a lactation consultant with Masters-level training was delegated to supervise the midwives and nurses on the maternity ward. Several practices with a strong evidence base for improving breastfeeding practices, such as early skin-to-skin contact (25), rooming-in (26), and allowing formula use only with a medical order from the pediatrician, were instituted. Finally, CMC's electronic medical record system was supplemented with an electronic version of the "newborn flow sheet," which had previously been hardcopy only. This aimed specifically to track feeding practices and to collect all data for better analysis.

The third phase of the intervention centered on patient education, a crucial component for behavior change given the baseline extremely low rates of EBF among the patient population. This phase included the addition of breastfeeding information into CMC's prenatal education classes; creation of promotional materials inside patient rooms (e.g., as inspirational messages on bathroom mirrors) and on take-home items (e.g., babies' bibs and hats); better documentation and standardization of prenatal and postnatal breastfeeding consultations by healthcare providers; detailed charting of breastfeeding practices at discharge; and the establishment of in-person and phone-based resources for new mothers postdischarge. Lastly, promotional campaigns at the hospital and online (via CMC's website and Facebook page) have sought to raise the profile of breastfeeding among patients and providers.

\section{Data Collection and Measures}

Qualitative data regarding the BFHI process were collected through iterative reflection sessions and semistructured interviews with core stakeholders who led implementation efforts, both at JHI and CMC. Breastfeeding data from 2,002 newborns delivered from July 2015 to February 2018 at CMC were examined to determine the overall impact of the collaborative BFHI process on monthly EBF rates. This period includes four months prior to intervention ("Lead-In"), the four-month window encompassing the bulk of the intervention period ("Intervention"), and 24 months of post-intervention follow-up ("FollowUp"). The EBF data are drawn from de-identified, aggregated hospital tracking data that include all live births during this time period.

For the purpose of this analysis, "exclusively breastfed" implies that mother and infant completed their entire inpatient stay (from birth to discharge, typically 2-4 days after birth) without the use of supplemental formula or other non-breastmilk liquids such as water or tea. Accordingly, monthly EBF rates were calculated as the number of "exclusively breastfed" infants divided by the number of total live births. Exclusion criteria were determined by $\mathrm{CMC}$ pediatricians and included infants managed in the intensive care setting, twin births, and infants for whom formula use was medically indicated. 


\section{Data Analysis}

Analysis of EBF data was performed via interrupted time series analysis with multiple treatment periods following a first-order autoregressive process. The ITSA command suite in STATA Version 15 software was utilized $(27,28)$. For the purposes of this data analysis, the "Intervention" was defined as the period between November 1, 2015, and February 29, 2016, during which the majority of the Phase 1-3 activities occurred.

\section{Ethical Considerations}

EBF and live birth rates were collected via de-identified hospital reporting data, and independently validated and audited by a third party through random, direct, on-site chart reviews. Data were stored in a HIPAA-compliant portal. Data collection procedures and protocols were reviewed by the Johns Hopkins Medicine Office of Human Subjects Research Institutional Review Board (application \#IRB00155989), and determined to not constitute human subjects research under DHHS or FDA regulations.

\section{Results}

The results of the interrupted time series analysis are shown in Fig. 1. In the first month for which data were collected (July 2015), the EBF compliance rate was $2.40 \%$. The baseline mean EBF compliance rate during the "Lead-In" period (July 2015 through October 2015, prior to most BFHI activities) was 3.33\% (95\% confidence interval [Cl]: 1.60,5.06). There was a non-significant decreasing trend in the EBF compliance rate during the "Lead-In" period of -0.37 percentage points per month $(p=0.54,95 \% \mathrm{Cl}:-1.58$, 0.84).

During the "Intervention" period (November 2015 through February 2016, in which the bulk of BFHI interventions occurred), the monthly change in EBF compliance was non-significantly greater than the "Lead-In" (pre-intervention) trend by +4.59 percentage points per month $(p=0.16,95 \% \mathrm{Cl}:-2.00,11.17)$. Mean EBF compliance during the "Intervention" period was $18.80 \%$ (95\% Cl: 8.30, 29.30).

During the "Follow-Up" period (March 2016 through February 2018, after the bulk of BFHI interventions were completed), the monthly rate of change of EBF was lower relative to the "Intervention" period trend by -2.59 percentage points per month $(p=0.39,95 \% \mathrm{Cl}=-8.67,3.49)$ but was statistically significantly greater than the trend during the baseline "Lead-In" period by +2.00 percentage points per month $(\mathrm{p}<$ $0.006,95 \% \mathrm{Cl}: 0.61,3.39)$. The overall mean EBF compliance rate during the "Follow-Up" period was $42.38 \%$ (95\% Cl: $36.62,48.14)$. In the final month for which data were collected (February 2018), the EBF compliance rate was $49.00 \%$.

Hence, in considering the overall impact of the BFHI process (comparing the post-intervention "Follow-Up" period to the pre-intervention "Lead-In" period), the intervention resulted in a statistically significant increase in the monthly rate of change in EBF compliance of +2.00 percentage points per month and an overall increase in EBF compliance, from $2.40 \%$ in the first month of data collection to $49.00 \%$ in the last month of data collection. Graphical and numerical comparisons of practices in the three periods are presented in Fig. 1 and Table 2. 
Table 2

Comparison of exclusive breastfeeding compliance before, during, and after a series of institutional interventions aimed at promoting Baby-Friendly Hospital practices

\begin{tabular}{|llll|}
\hline Mean EBF Rate (95\% confidence interval) & $\begin{array}{l}3.33 \%(1.60, \\
\text { (18.80\% }(8.30,\end{array}$ & $\begin{array}{l}42.38 \%(36.62, \\
29.30)\end{array}$ & $48.14)$ \\
\hline $\begin{array}{l}\text { Monthly change in EBF rate (95\% } \\
\text { confidence interval) }\end{array}$ & $\begin{array}{l}-0.37 \\
\text { percentage } \\
\text { points }\end{array}$ & $\begin{array}{l}+4.22 \\
\text { percentage } \\
\text { points }\end{array}$ & $\begin{array}{l}+1.63 \\
\text { percentage } \\
\text { points }\end{array}$ \\
& $(-1.58,0.84)$ & $(-1.95,2.31)$ & $(0.96,2.31)$ \\
\hline $\begin{array}{l}\text { Difference in Monthly change in EBF rate } \\
\text { (95\% confidence interval) }\end{array}$ & Reference & $\begin{array}{l}4.59(-2.00,11.2 ; \\
\mathrm{p}=0.164)\end{array}$ & $\begin{array}{l}2.00(0.61,3.39 ; \\
\mathrm{p}=0.006)\end{array}$ \\
\hline
\end{tabular}

\section{Note}

The dashed vertical lines represent the time period from November 1, 2015, to February 29, 2016 during which the majority of BFHI interventions occurred (i.e., Phases 1-3).

\section{Discussion}

This study uses an interrupted time series methodology to demonstrate the impact of implementing the BFHI package among an initially EBF-averse patient population. The absolute rate of EBF at hospital discharge increased from 2.4-49.0\% from the first month to the final month of data recorded, and the above analysis conveys the improved trends in EBF practices over time. This analysis showed overall increases in the adoption of EBF during both the "Intervention" and "Follow-Up" periods, as well as a statistically significant change in the monthly increase in EBF adoption when compared to baseline. Moreover, these observed behavioral trends in the adoption of EBF practices persisted up to two years following the initial intervention period.

Implementation of the full BFHI package is a complex intervention. Each of the WHO/UNICEF-validated "10 Steps" involves multiple discrete actions and changes that require buy-in from hospital administration, healthcare staff, and patients. Using Powell et al.'s framework from the implementation science literature, this process included implementation strategies related to planning, education, restructuring, and quality management (29). A timeline of when specific interventions were implemented is available in Additional File 1; however, many of these changes were gradual, and the effect of the BFHI package was likely cumulative over time.

From the CMC team's perspective, a key factor in the successful implementation of BFHI and the resulting increase in EBF rates was the engagement of a core team of care providers. This multidisciplinary team included midwives, nurse educators, pediatricians, and obstetricians. They served as "champions" for the $\mathrm{BFHI}$ process and led the process of policy revision and implementation of new procedures. The CMC team cited pediatricians as very influential in women's decisions to breastfeed, a finding that is supported 
by several previous studies in Lebanon $(7,11)$. Hospital administration and management were also engaged in the BFHI process, allowing for institution-wide reforms and unified messaging. Positive feedback from future mothers and their families regarding CMC's BFHI improvements and the surrounding promotional campaign has provided the momentum for the process to continue.

Several challenges arose during BFHI implementation, many of which reflect cultural barriers to breastfeeding that persist in Lebanon. For practices such as skin-to-skin contact after birth, adoption of such practices by women occurred quickly, demonstrating that successful behavior change is possible. However, many CMC patients, family members, and even occasionally outpatient healthcare providers still expressed doubt that breastmilk is nutritionally sufficient for newborns. Some mothers also resisted the rooming-in policy, expressing the need to rest while their infant is cared for in a nursery. The CMC team addressed these challenges by adopting patient-centered communication techniques and focusing on the benefits of breastfeeding for both mother and newborn. Women were empowered to make their own decisions and were supported accordingly. Continuing educational outreach, particularly on the sufficiency of breastmilk for infant nutrition, will be important to address these challenges and further increase EBF rates in the future.

Another challenge in implementing this initiative is reflective of the fact that $\mathrm{CMC}$ employs a combination of full-time clinicians in addition to part-time providers from the community. For those part-time providers (who primarily practice outside the hospital but bring their patients to $\mathrm{CMC}$ for delivery), it has been more difficult to become engaged in the Phase 2 activities aimed at changing clinical practices, and accordingly, EBF adoption has been slower in this group. Further targeted outreach to these providers is planned.

Finally, an ongoing audit of the long-term breastfeeding practices of new mothers in the outpatient setting (i.e., following discharge from the hospital) is still actively being developed. Although our study and others show that BFHI can be successful in increasing EBF in early infancy, maintaining EBF through the recommended six months of age remains a challenge $(7,30)$. For example, in their BFHI trial in Saudi Arabia, Mosher et al. showed a significant decrease in EBF rates at six months among both the intervention (BFHI hospital) and control (non-BFHI hospital) groups (21). Systematic reviews have shown the importance of ongoing postpartum support to mothers for EBF continuation $(18,31)$, corresponding to Step 10 in the BFHI package. In Lebanon, a previous study has shown the positive impact of a telephone support hotline on EBF rates (8), an encouraging result for the hotline set up by CMC as a part of this study. A recently-completed randomized controlled trial at two other hospitals in Lebanon has shown the effectiveness of a comprehensive package of professional and peer postpartum support on EBF knowledge and practice $(30,32)$. Looking forward, CMC will evaluate such initiatives for their utility in maintaining breastfeeding practices after new mothers have left the inpatient setting.

\section{Strengths and Limitations}

This study has a number of important strengths. Although data were analyzed retrospectively, they were collected continuously throughout the initiative, which allows for evaluating trends over time rather than 
mere pre/post comparisons. Interrupted time series is a strong quasi-randomized study design when the use of a control group is not feasible $(33,34)$. In addition, this study's use of patient data allowed for an objective measure (as recorded by healthcare providers) of EBF practice, compared to mothers' self-report in post-discharge surveys used in other studies (21). The EBF data have been strictly audited as a part of CMC's partnership with $\mathrm{JHI}$ and validated by the quality department at CMC. Finally, the study sample size was large, with 2,002 live births included.

It is also important to acknowledge several limitations of this study. It was conducted retrospectively and in a single center, meaning that no control group was present; however, the interrupted time series analysis is a methodologically appropriate means for addressing this limitation (33). In addition, because de-identified hospital reporting data were used, we were not able to measure demographic information of mothers or infants and account for any demographic shifts over time. This is an important avenue for further research, which could include prospective enrollment of participants and collection of individuallevel data, in order to analyze trends among specific subgroups of mothers. Finally, our outcome measure in the evaluation of this program is the EBF rate at discharge (typically 2-4 days after birth), which, although clearly associated with EBF rates at one and six months of age (7), is admittedly a surrogate marker for such practices. Future studies at CMC should include post-discharge follow-up through six months of age, to allow for comparison with national EBF trends.

\section{Conclusions}

In Lebanon and elsewhere, hospital-based prenatal and early postnatal interactions are a crucial entry point for behavior change regarding breastfeeding $(7,20)$. Although the impact of BFHI certification in and of itself has been questioned, it is clear that specific evidence-based interventions within the BFHI package can lead to meaningful improvements in breastfeeding practice $(20,35)$. The results of our study further support the importance of the hospital environment in improving EBF rates, particularly in a cultural and clinical setting with low baseline compliance.

The CMC experience highlights several important factors for healthcare institutions considering the implementation of BFHI standards. First is the use of a multidisciplinary team of care providers who can champion institutional baby-friendly practices while also dispelling misconceptions through sustained educational outreach to clinical providers and hospital staff. Next, clinician-led promotional efforts centered on prenatal education on EBF for future mothers and their families, as well as regular tracking of data to measure the adoption of breastfeeding practices in new mothers, is critical. Finally, the creation of programs designed to educate and support new mothers in sustaining such breastfeeding practices in the outpatient setting is an essential component. Upholding the principles outlined in the BFHI is a complex process that involves changes to hospital policies, staff practices, and physical infrastructure. However, with an appropriate institutional commitment, as well as staff engagement across many levels of patient care, it can be an achievable goal, leading to meaningful improvements in breastfeeding practice. 


\section{List Of Abbreviations}

$\mathrm{EBF}=$ exclusive breastfeeding

WHO = World Health Organization

BFHI = Baby-Friendly Hospital Initiative

$\mathrm{CMC}=$ Clemenceau Medical Center

$\mathrm{JCl}=$ Joint Commission International

$\mathrm{JHI}=$ Johns Hopkins Medicine International

\section{Declarations}

Ethics approval and consent to participate: Data collection procedures and protocols were reviewed by the Johns Hopkins Medicine Office of Human Subjects Research Institutional Review Board (application \#IRB00155989), and determined to not constitute human subjects research under DHHS or FDA regulations.

Consent for publication: Not applicable.

Availability of data and materials: The datasets used and/or analyzed during the current study are available from the corresponding author on reasonable request.

Competing interests: The authors declare that they have no competing interests.

Funding: This project was facilitated and funded through a collaboration between Clemenceau Medical Center and Johns Hopkins Medicine International (JHI). JHI is a subsidiary of the Johns Hopkins University and the Johns Hopkins Health System.

This project was made possible in part by the Johns Hopkins Institute for Clinical and Translational Research (ICTR) which is funded in part by Grant Number UL1 TR001079 from the National Center for Advancing Translational Sciences (NCATS), a component of the National Institutes of Health $(\mathrm{NIH})$, and $\mathrm{NIH}$ Roadmap for Medical Research. Its contents are solely the responsibility of the authors and do not necessarily represent the official view of the Johns Hopkins ICTR, NCATS, or NIH.

Authors' contributions: JEG, RH, and MS led implementation of the intervention at Clemenceau Medical Center. HB, WB, NR, JB, KB provided scientific, administrative, and leadership support from Johns Hopkins, and made substantial contributions to the program's evaluation plan. AC and RS led the methodological and analytical plan for this manuscript, as well as initial manuscript preparation and parts of the statistical analysis. JW was responsible for supervising and evaluating the statistical 
analysis of the data. All authors were involved in subsequent edits to the manuscript and revised/approved the final manuscript.

Acknowledgements: The study team wishes to thank the multidisciplinary efforts of multiple clinical and non-clinical providers within Clemenceau Medical Center. Special thanks as well to Drs. Karen Haller and Charles Wiener from the executive research committee of Johns Hopkins Medicine International, and to the Johns Hopkins Post-Baccalaureate Premedical Program for their support of the work of Ms. Clermont

\section{References}

1. American Academy of Pediatrics. Breastfeeding and the use of human milk. Pediatrics. 2012 Mar;129(3):e827-841.

2. Victora CG, Bahl R, Barros AJD, França GVA, Horton S, Krasevec J, et al. Breastfeeding in the 21 st century: epidemiology, mechanisms, and lifelong effect. The Lancet. 2016 Jan;387(10017):475-90.

3. Rollins NC, Bhandari N, Hajeebhoy N, Horton S, Lutter CK, Martines JC, et al. Why invest, and what it will take to improve breastfeeding practices? The Lancet. 2016 Jan;387(10017):491-504.

4. World Health Organization. Infant and young child nutrition: Global strategy on infant and young child feeding [Internet]. 2002 [cited 2017 Oct 29]. Available from: http://apps.who.int/gb/archive/pdf_files/WHA55/ea5515.pdf.

5. American College of Obstetricians and Gynecologists. Committee Opinion Number 658: Optimizing Support for Breastfeeding as Part of Obstetric Practice [Internet]. 2016 [cited 2019 Mar 17]. Available from: https://www.cdc.gov/breastfeeding/pdf/optimizing-support-for-breastfeeding-as-part-ofobstetric-practice.pdf.

6. Republic of Lebanon Ministry of Public Health. Hasbani and the UNICEF Launched the National Breastfeeding Campaign [Internet]. 2018 [cited 2020 Apr 4]. Available from: http://www.moph.gov.lb.

7. Batal M, Boulghourjian C, Abdallah A, Afifi R. Breast-feeding and feeding practices of infants in a developing country: a national survey in Lebanon. Public Health Nutr. 2006 May;9(3):313-9.

8. Hamade H, Chaaya M, Saliba M, Chaaban R, Osman H. Determinants of exclusive breastfeeding in an urban population of primiparas in Lebanon: a cross-sectional study. BMC Public Health. $2013 \mathrm{Jul}$ 31;13:702.

9. Nabulsi M. Why are breastfeeding rates low in Lebanon? A qualitative study. BMC Pediatr. 2011 Aug;30:11:75.

10. Hamade H, Naja F, Keyrouz S, Hwalla N, Karam J, Al-Rustom L, et al. Breastfeeding knowledge, attitude, perceived behavior, and intention among female undergraduate university students in the Middle East: the case of Lebanon and Syria. Food Nutr Bull. 2014 Jun;35(2):179-90.

11. Al-Sahab B, Tamim H, Mumtaz G, Khawaja M, Khogali M, Afifi R, et al. Predictors of breast-feeding in a developing country: results of a prospective cohort study. Public Health Nutr. 2008 Dec;11(12):1350-6. 
12. Osman H, El Zein L, Wick L. Cultural beliefs that may discourage breastfeeding among Lebanese women: a qualitative analysis. Int Breastfeed J. 2009 Nov 2;4:12.

13. Akik C, Ghattas H, Filteau S, Knai C. Barriers to breastfeeding in Lebanon: A policy analysis. J Public Health Policy. 2017 Aug;38(3):314-26.

14. World Health Organization. Baby-friendly Hospital Initiative [Internet]. 2017 [cited 2017 Oct 29]. Available from: http://www.who.int/nutrition/topics/bfhi/en/.

15. WHO/UNICEF. Implementation Guidance: Protecting, promoting and supporting breastfeeding in facilities providing maternity and newborn services: the revised Baby-Friendly Hospital Initiative 2018 [Internet]. 2018. Available from: https://apps.who.int/iris/bitstream/handle/10665/272943/9789241513807-eng.pdf.

16. Bass JL, Gartley T, Kleinman R. World Health Organization Baby-Friendly Hospital Initiative Guideline and 2018 Implementation Guidance. JAMA Pediatr. 2019 Jan 1;173(1):93-4.

17. Labbok MH. Global baby-friendly hospital initiative monitoring data: update and discussion. Breastfeed Med Off J Acad Breastfeed Med. 2012 Aug;7:210-22.

18. Pérez-Escamilla R, Martinez JL, Segura-Pérez S. Impact of the Baby-friendly Hospital Initiative on breastfeeding and child health outcomes: a systematic review. Matern Child Nutr. 2016 Jul;12(3):402-17.

19. Howe-Heyman A, Lutenbacher M. The Baby-Friendly Hospital Initiative as an Intervention to Improve Breastfeeding Rates: A Review of the Literature. J Midwifery Womens Health. 2016 Feb;61(1):77102.

20. Gomez-Pomar E, Blubaugh R. The Baby Friendly Hospital Initiative and the ten steps for successful breastfeeding: a critical review of the literature. J Perinatol Off J Calif Perinat Assoc. 2018 Jun;38(6):623-32.

21. Mosher C, Sarkar A, Hashem AA, Hamadah RE, Alhoulan A, AlMakadma YA, et al. Self-reported breast feeding practices and the Baby Friendly Hospital Initiative in Riyadh, Saudi Arabia: prospective cohort study. BMJ Open [Internet]. 2016 Dec 16;6(12). Available from:

https://www.ncbi.nlm.nih.gov/pmc/articles/PMC5168637/.

22. Edwards G, Abdulali J, Kumar RR. Meeting the challenge: implementing the Baby Friendly Hospital Initiative in a culturally diverse country. Pract Midwife. 2011 Jun;14(6):12, 14-6.

23. Al Ghazal H, Rashid S, Ruf E. The Sharjah Baby-Friendly Campaign: A Community-Based Model for Breastfeeding Promotion, Protection, and Support. Breastfeed Med Off J Acad Breastfeed Med. 2015 Nov;10(9):437-41.

24. Ogrinc G, Davies L, Goodman D, Batalden P, Davidoff F, Stevens D. SQUIRE 2.0 (Standards for QUality Improvement Reporting Excellence): revised publication guidelines from a detailed consensus process. BMJ Qual Saf. 2016 Dec 1;25(12):986-92.

25. 10.1002/14651858.CD003519.pub4/abstract Moore ER, Bergman N, Anderson GC, Medley N. Early skin-to-skin contact for mothers and their healthy newborn infants. In: Cochrane Database of Systematic Reviews [Internet]. John Wiley \& 
Sons, Ltd; 2016. Available from:

http://onlinelibrary.wiley.com/doi/10.1002/14651858.CD003519.pub4/abstract.

26. 10.1002/14651858.CD006641.pub3/abstract

Jaafar SH, Ho JJ, Lee KS. Rooming-in for new mother and infant versus separate care for increasing the duration of breastfeeding. In: Cochrane Database of Systematic Reviews [Internet]. John Wiley \& Sons, Ltd; 2016. Available from:

http://onlinelibrary.wiley.com/doi/10.1002/14651858.CD006641.pub3/abstract.

27. StataCorp. Stata Statistical Software: Release 15. College Station. TX: StataCorp LLC; 2017.

28. Linden A. Conducting interrupted time series analysis for single and multiple group comparisons. Stata J. 2015 Jun 1;15:480-500.

29. Powell BJ, McMillen JC, Proctor EK, Carpenter CR, Griffey RT, Bunger AC, et al. A Compilation of Strategies for Implementing Clinical Innovations in Health and Mental Health. Med Care Res Rev MCRR. 2012 Apr;69(2):123-57.

30. Nabulsi M, Hamadeh H, Tamim H, Kabakian T, Charafeddine L, Yehya N, et al. A complex breastfeeding promotion and support intervention in a developing country: study protocol for a randomized clinical trial. BMC Public Health. 2014 Jan 15;14:36.

31. McFadden A, Gavine A, Renfrew MJ, Wade A, Buchanan P, Taylor JL, et al. Support for healthy breastfeeding mothers with healthy term babies. Cochrane Database Syst Rev. 2017;28:2:CD001141.

32. Nabulsi M, Tamim H, Shamsedine L, Charafeddine L, Yehya N, Kabakian-Khasholian T, et al. A multicomponent intervention to support breastfeeding in Lebanon: A randomized clinical trial. PLOS ONE. 2019 Jun;14(6):e0218467. 14(.

33. Kontopantelis E, Doran T, Springate DA, Buchan I, Reeves D. Regression based quasi-experimental approach when randomisation is not an option: interrupted time series analysis. BMJ. 2015 Jun;9:350:h2750.

34. Penfold RB, Zhang F. Use of interrupted time series analysis in evaluating health care quality improvements. Acad Pediatr. 2013 Dec;13(6 Suppl):38-44.

35. Brodribb W, Kruske S, Miller YD. Baby-friendly hospital accreditation, in-hospital care practices, and breastfeeding. Pediatrics. 2013 Apr;131(4):685-92.

\section{Figures}




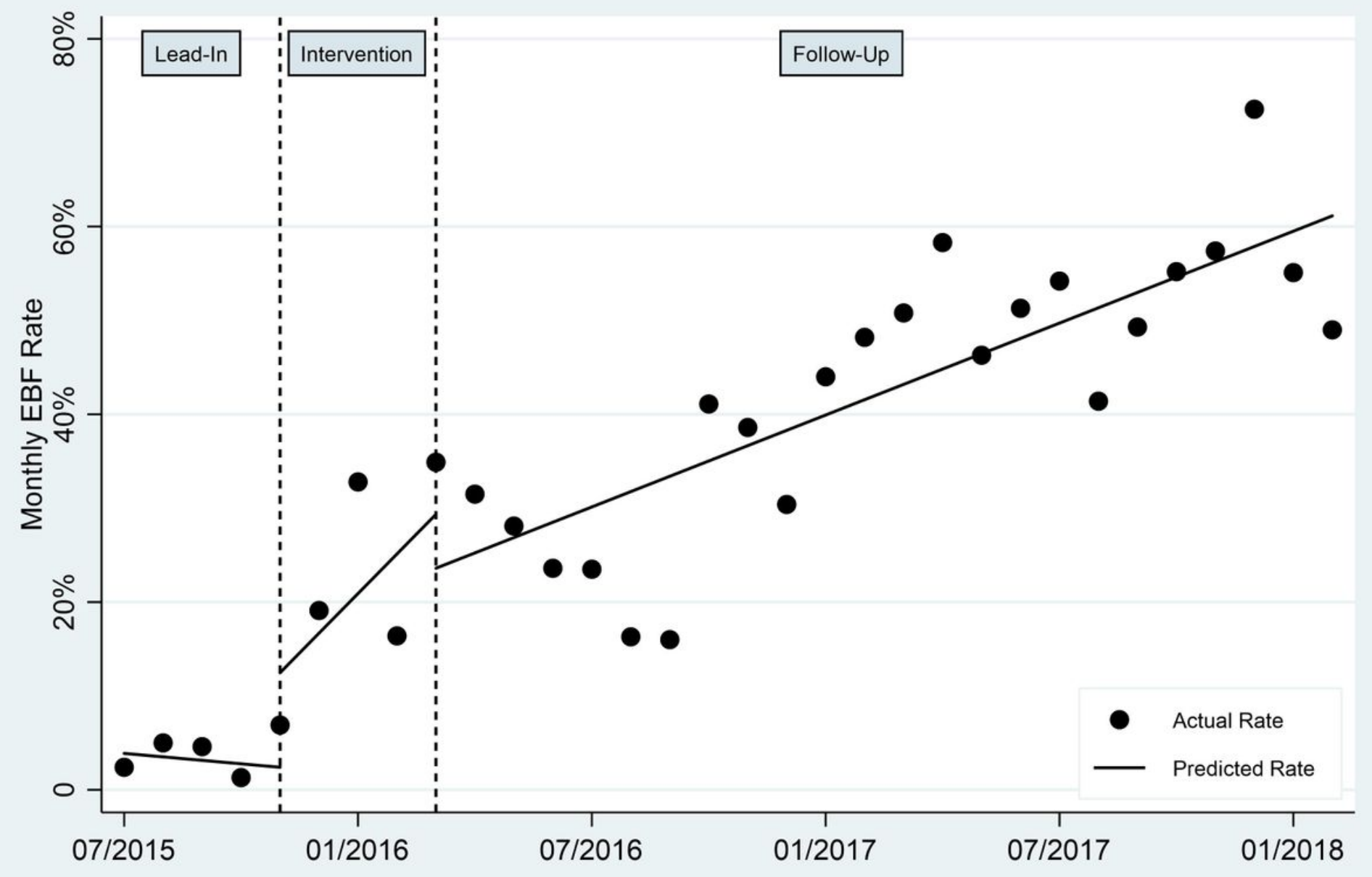

Figure 1

Interrupted Time Series Analysis of Exclusive Breastfeeding Rates at CMC Note: The dashed vertical lines represent the time period from November 1, 2015, to February 29, 2016 during which the majority of BFHI interventions occurred (i.e., Phases 1-3).

\section{Supplementary Files}

This is a list of supplementary files associated with this preprint. Click to download.

- AdditionalFile12020.pdf 